\title{
Balanceamento Automático de Metas para Estabilização de Indicadores de Saúde na Atenção Básica
}

\author{
Nécio de Lima Veras ${ }^{1}$, Windson Viana ${ }^{2}$, Mizael Lima da Silva ${ }^{1}$, \\ Ely Bezerra Silva Júnior ${ }^{1}$ \\ ${ }^{1}$ Instituto Federal do Ceará (IFCE) - Tianguá - CE - Brasil \\ ${ }^{2}$ Departamento de Computação - Universidade Federal do Ceará (UFC) \\ Fortaleza - CE - Brasil \\ necio.verasdifce.edu.br, windsonegreat.ufc.br \\ mizaellimsilegmail.com, elyjunior@foxinline.com
}

\begin{abstract}
The planning of goal-oriented services for health indices promotes the access and the quality in providing these services. Automatic balancing of goals aims to improve the indicators, try to approximate them to the values initially planned, especially when health managers are dealing with inconstant scenarios. In this context, this paper formalises the automatic goal balancing, incorporating it into a tool. This software is capable of simulate contingent scenarios regarding initial deviation plans. With a set of simulations inspired in realistic scenarios, we find results statistically significant $(p<0.05)$ that offer satisfactory and positive values for our proposal, suggesting the approach would work in a real environment properly.
\end{abstract}

Resumo. O planejamento de serviços orientados por metas para indicadores de saúde promove a melhoria do acesso e da qualidade da oferta desses serviços. Um balanceamento automático de metas objetiva melhorar a aproximação dos indicadores em relação ao valor originalmente planejado em cenários inconstantes. Neste contexto, este artigo formaliza o balanceamento automático de metas, incorporando-o em uma ferramenta capaz de simular cenários contingentes que desviam de um plano inicial. Os resultados estatísticos do uso da proposta, a partir de simulações inspiradas em cenários realistas, são satisfatórios e ofereceram valores positivos $(p<0,05)$, sugerindo que a abordagem poderia funcionar corretamente em um ambiente real.

\section{Introdução}

No Brasil, o ministério da saúde estruturou o Programa de Melhoria do Acesso e da Qualidade na Atenção Básica (PMAQ-AB) [BRASIL 2012]. Idealizou-se, dentre outras coisas, a utilização de parâmetros de comparação entre as equipes de saúde considerando diferentes realidades. Outro ponto foi o melhoramento incremental e contínuo de indicadores de acesso e de qualidade relacionados com a oferta de serviços de saúde. Estão envolvidos no processo: o planejamento orientado pela busca de resultados e o monitoramento e acompanhamento de indicadores. Tendo em vista que a unidade de avaliação do PMAQ é a equipe de saúde, o cálculo de indicadores objetiva mensurar o desempenho das equipes em relação ao acesso e a qualidade dos serviços oferecidos. 
No âmbito da informática em saúde, ainda existem poucos projetos visando tornar o planejamento orientado por metas aplicável no cotidiano de profissionais de saúde; mais especificamente em relação ao desenvolvimento de ferramentas que possibilitem automatizar, otimizar e monitorar indicadores de saúde. Na literatura, existem trabalhos que propõem auxiliar as equipes de saúde em processos ligados à implantação do PMAQ [Oliveira et al. 2012] [Silva et al. 2012]. Entretanto, de acordo com o que foi encontrado, nenhuma das soluções usa a inteligência artificial como estratégia para a geração de soluções inovadoras, como pode ser encontrado em pesquisas que focam o ambiente hospitalar [Isern et al. 2010], [Cardoso and al 2014], [Braun et al. 2005], [Vermeulen et al. 2009] e [Wiesman et al. 2006].

O presente trabalho propõe formalizar o balanceamento automático de metas para o problema da alocação da oferta de serviços de saúde em uma agenda dinâmica capaz de atuar no planejamento de atendimentos orientado por metas. O trabalho é uma continuação da abordagem de [Veras et al. 2018] que otimizou a alocação de serviços de saúde associados à Atenção Básica por meio de técnicas de inteligência computacional. Para tanto, neste artigo, foi avaliado o uso da estratégia e comparado com resultados gerados sem o uso do balanceamento proposto. O restante deste artigo apresenta a metodologia de pesquisa, as formulações matemáticas e discute a avaliação com os resultados.

\section{Metodologia}

Este trabalho é uma intervenção de natureza quantitativa, um estudo in vitro executado em um ambiente controlado. A pesquisa foi dividia em quatro estágios. No primeiro, foi investigado como atendimentos de saúde são realizados em unidades básicas de saúde e, para isso, fez-se observações sem intervenções. No segundo estágio, realizou-se buscas bibliográficas com o contexto abordado. No terceiro passo, foi formulado o problema do planejamento de atendimentos orientado por metas e do balanceamento automático dessas metas para ser incorporado em uma ferramenta computacional. Finalmente, no quarto estágio, avaliou-se o impacto da ferramenta por meio de simulações computacionais em um ambiente controlado semelhante à realidade de uma equipe de saúde.

\subsection{Coleta de dados}

A coleta de dados deu-se a partir de observações e entrevistas semi-estruturas com profissionais de enfermagem de quatro diferentes unidades básicas de saúde de um município brasileiro. Foram coletados dados quantitativos e anonimizados sobre variáveis populacionais e metas coletivas da equipe de saúde (Tabela 2).

\subsection{Simulações computacionais}

Todas as simulações foram realizadas em um único computador e os resultados obtidos foram originados a partir da média das soluções encontradas em um universo de trinta execuções para os cenários construídos. Para tanto, foram criados dois cenários $(\mathrm{C} 1$ e C2) com dois grupos de testes. O primeiro cenário $(\mathrm{C} 1)$ é um cenário de controle que visa mostrar a independência dos cálculos da abordagem (descritos nas seções 3 e 4.1) entre agendas planejadas e simuladas. É denominado de controle pois qualquer mudança na geração de um planejamento não afeta o resultado final.

O segundo cenário (C2) estabelece uma probabilidade individual para a ocorrência de eventos contingentes associados à oferta de serviços de saúde, aproximando as 
simulações a um ambiente realista. Neste sentido, consultou-se dez profissionais de três equipes de saúde de um município brasileiro, solicitando a enumeração de eventos rotineiros capazes de atrapalhar a execução de serviços de saúde, bem como, valores individuais para as probabilidades de ocorrência desses eventos. Ao final, foram sugeridas probabilidades uniformes com valores globais variando entre $5 \%$ e $20 \%$, dependendo do serviço ofertado e do evento associado. Além disso, perguntou-se também se, diante da ocorrência de algum evento e da impossibilidade de realizar um atendimento, um outro serviço poderia ser ofertado substituindo o serviço ora planejado. Com as respostas dos profissionais foi possível montar um plano de substituição de serviços não realizados e incorporá-los nas simulações, nomeados de "com serviços alternativos" e "sem serviços alternativos".

\section{Atendimentos orientados por metas: um problema de otimização}

Dado que uma equipe de saúde oferta diversos serviços e que estes modificam os valores de um ou mais indicadores de saúde, então a tarefa de planejar uma agenda de atendimentos de forma a otimizar os valores para indicadores distintos é complexa, principalmente quando existe uma pactuação da equipe com a gestão municipal estabelecendo metas de longo prazo para os indicadores.

\subsection{Formulação do problema de otimização orientado por metas}

A atenção básica de um município $M$ é composta por $n$ equipes de saúde $E$, definidos como $M=\left\{E_{1}, E_{2}, \ldots, E_{n}\right\}$. Cada equipe é formada por um conjunto de $m$ profissionais dado por $E=\left\{P_{1}, P_{2}, \ldots, P_{m}\right\}$. Existe um conjunto $S$ de $o$ serviços de saúde que podem ser ofertados pelas equipes, dado por $S=\left\{S_{1}, S_{2}, \ldots, S_{o}\right\}$, sendo que uma oferta individual pode ser menor ou igual a $o$. Vale ressaltar que cada profissional $P_{j} \in E$ pode estar associado a um ou vários serviços pertencente ao conjunto $S$. O conjunto dos custos representa o tempo necessário para que um determinado serviço seja executado, representado como $C=\left\{C_{1}, C_{2}, \ldots, C_{p}\right\}$, onde $p \leq o$. Um serviço qualquer $S_{y} \in S$ ao ser alocado em uma agenda de atendimentos de um profissional $P_{j} \in E$ pode contribuir com a valoração de um ou mais indicadores de saúde.

A construção de uma estrutura computacional de uma agenda dinâmica, deve considerar que um dia $d$ de trabalho possui instantes $k$ definidos como a sequência $k=(1,2, \ldots, t)$, onde $t$ representa o limite superior de tempo para um dia de trabalho. Um profissional $P_{j} \in E$ em um instante $k$ de um dia $d$ realiza o serviço $S_{y} \in S$. A função trab indica o custo $C_{z} \in C$ de um serviço $S_{y} \in S$ executado por um profissional $P_{j} \in E$ em um instante $k$ de um dia $d$. Assim, o custo de trabalho de um profissional $P_{j} \in E$ para um serviço $S_{y} \in S$ em um período de tempo constituído por no máximo max dias e $t$ instantes é denotado por $r$, dado por:

$$
r=\sum_{d=1}^{\max } \sum_{k=1}^{t} \operatorname{trab}\left(d^{k}, P_{j}, S_{y}\right)
$$

É importante atestar que cada serviço $S_{y} \in S$ está relacionado a um conjunto indicadores de saúde, dado por $I=\left\{I_{1}, I_{2}, \ldots, I_{w}\right\}$ e que, cada indicador possui suas próprias variáveis associadas com algum dado populacional ou sobre a oferta de um serviço específico. O conjunto $T=\left\{T_{1}, T_{2}, \ldots, T_{o}\right\}$ estabelece metas para os serviços prestados 
no contexto de uma agenda. Para cada elemento $T_{y} \in T$ existe um serviço $S$ associado, formalmente definido como $\forall T_{y} \in T \exists S_{y}$. Assim, cada meta $T_{y} \in T$ é um valor desejado para o serviço $S_{y} \in S$. A demanda social (seção 3.2) de um serviço $S_{y} \in S$ para um profissional $P_{j} \in E$ é dada por $D_{P_{j}}^{S_{y}}$ e é calculada a partir de uma meta $T_{y} \in T$ com as variáveis associadas aos indicadores pertencentes à $S_{y} \in S$. A Seção 3.2 apresenta uma ilustração de demanda social.

Define-se que uma agenda semanal $G_{s}$ associada a um profissional $P_{j} \in E$ é representada como uma matriz composta por $l$ linhas e $c$ colunas que indicam a alocação de um serviço $S_{y} \in S$ na agenda, onde $l$ representa os dias e $c$ a unidade de tempo de execução do serviço, formalmente definida como:

$$
G_{s}^{P_{j}}=\left(S_{y a b}\right)_{l \times c}=\left(\begin{array}{cccc}
S_{y 11} & S_{y 12} & \ldots & S_{y 1 c} \\
S_{y 21} & S_{y 22} & \ldots & S_{y 2 c} \\
\vdots & \vdots & \vdots & \vdots \\
S_{y l 1} & S_{y l 2} & \ldots & S_{y l c}
\end{array}\right), \quad \text { onde } l \leq t \quad e \quad c \leq 5
$$

Uma agenda mensal $G_{m s}$ de um profissional $P_{j} \in E$ pode ser visualizada como um conjunto de até cinco agendas semanais, dado por $G_{m s}^{P_{j}}=\left\{G_{s_{1}}^{P_{j}}, G_{s_{2}}^{P_{j}}, G_{s_{3}}^{P_{j}}, G_{s_{4}}^{P_{j}}, G_{s_{5}}^{P_{j}}\right\}$.

Para o modelo proposto, seguindo recomendações do PMAQ [BRASIL 2012], observou-se que (1) certos indicadores são influenciados pela realização dos serviços de uma agenda e podem ser calculados a partir dos serviços alocados na agenda; (2) outros indicadores não sofrem nenhuma alteração em seus valores por conta dos serviços de uma agenda; e (3) existem indicadores que influenciam no planejamento da alocação de serviços de uma agenda e, portanto são determinantes para a valoração das quantidades dos serviços a serem alocados. Como resultado, a partir de um universo comum de indicadores de saúde foi extraído um subconjunto de indicadores ligados a seguinte oferta de serviços em saúde: Atendimento de Pré-natal; Prevenção do câncer ginecológico; Visitas domiciliares; Consulta de puericultura; Consulta médica; Consulta de Hipertensos e Diabéticos; Ação coletiva de escovação dental supervisionada; Consulta Odontológica; Consulta Odontológica à Gestante; Consulta de enfermagem e Vigilância (tuberculose e hanseníase).

O formulação proposta considera que as metas $T$ são individualizadas conforme o serviço ofertado. A ideia é definir metas locais, calcular as demandas para estimar o quantitativo dos serviços ofertados para um profissional e alocá-los em uma agenda para posterior avaliação de qualidade da agenda da equipe gerada. O volume (em horas) de trabalho de um profissional em relação a um serviço $S_{y} \in S$ no contexto de $G_{m s}^{P_{j}}$ indica quantas unidades de tempo um serviço foi alocado na agenda, representado por $V_{P_{j}}^{S_{y}}$. A partir disso, é possível determinar a qualidade de $G_{m s}^{P_{j}}$ com a seguinte equação:

$$
\begin{gathered}
Q_{G_{m s}^{P_{j}}}=\sum_{i=1}^{\text {Servs }} \frac{V_{P_{j}}^{S_{i}}}{D_{P_{j}}^{S_{i}}}-P 1-P 2-P 3 \text { tal que: } \\
P 1=\left(\sum_{d=1}^{D \text { Servs }^{+}} \frac{V_{P_{j}}^{S_{d}}}{D_{P_{j}}^{S_{d}}}\right) \times 2 ; P 2=\left(\sum_{l=1}^{D \text { Servs }^{-}} \frac{D_{P_{y}}^{S_{l}}}{V_{P_{j}}^{S_{l}}}\right) \times 6 ; P 3=\left(\sum_{q=1}^{D \operatorname{Servs}^{n}} \frac{D_{P_{j}}^{S_{q}}}{100}\right) \times 10 ;
\end{gathered}
$$


É importante estabelecer que $\forall S_{i}, S_{j}, S_{l} \in G_{m s}^{P_{j}}$ e $\forall S_{q} \notin G_{m s}^{P_{y}}$ temos que:

- Servs é a contagem dos serviços não repetidos de $G_{m s}^{P_{y}}$,

- DServs ${ }^{+}$é a contagem dos serviços alocados em $G_{m s}^{P_{j}}$ que estão acima de $D_{P_{j}}^{S_{d}}$,

- DServs ${ }^{-}$é a contagem dos serviços alocados em $G_{m s}^{P_{j}}$ que estão abaixo de $D_{P_{j}}^{S_{l}}$ e

- $D S e r v s^{n}$ é a contagem dos serviços NÃO alocados em $G_{m s}^{P_{j}}$, mas que pertencem ao conjunto $S$ associado a um dos profissionais de $P$.

Desse modo, a função de qualidade (Equação 3) avalia o grau de cumprimento dos respectivos serviços em relação às suas demandas. A primeira soma agrupa esse grau para todos os serviços alocados na agenda considerando a relação entre volume de alocação e demanda para os serviços. As somas $P 1, P 2$ e $P 3$ funcionam como penalidades aos serviços desbalanceados em relação as suas demandas. O serviço cujo volume está acima de sua demanda $(P 1)$ é penalizado com peso dois, enquanto que o serviço abaixo da demanda $(P 2)$ sofre uma punição mais rígida com peso seis. O último somatório $(P 3)$ aplica uma penalidade de peso dez ao serviço que deveria ter sido alocado na agenda, mas não foi. Os pesos fixados foram os mais adequados encontrados durantes os testes apresentados em [Veras 2015]. Portanto, a qualidade média das agendas mensais de uma equipe $E_{e} \in M$ é calculada por:

$$
Q_{G_{m s}}^{E_{e}}=\frac{\sum_{x=1}^{m} Q_{G_{m s}^{P_{x}}}}{m}, \text { onde } P_{x} \in E_{e}
$$

\subsection{Cálculo das demandas sociais}

O cálculo das demandas sociais para cada serviço ofertado está relacionado com (a) variáveis sociais, (b) custos para execução dos serviços e (c) metas individuais traçadas pela equipe de saúde. Um exemplo de variáveis sociais para a oferta do serviço "Prénatal" são: número de atendimentos durante o pré-natal e número de gestantes cadastradas.

Um profissional $P_{j} \in E$ pode ser associado a um ou mais serviços. Esse profissional em um dado instante $k$ de um dia de trabalho executa apenas um serviço e fica alocado nele durante um certo período de tempo representado por um custo. A Tabela 1 mostra uma configuração de participação dos profissionais de uma equipe nos serviços ofertados e o custo fixo para as suas execuções. Considerou-se o conjunto de profissionais de uma equipe formado por Enfermeiros, Médicos e Odontólogos e Agentes Comunitários de Saúde (ACS), no entanto, o planejamento de uma agenda de atendimentos não contempla os ACS's. De forma a ilustrar um cálculo para demandas sociais, $D_{1}$ refere-se ao Atendimento de Pré-Natal. Para o cálculo é considerado o custo de um atendimento de 30 minutos (durante um instante $k$ serão realizados dois atendimentos). O objetivo foi estipular o número de atendimentos para pré-natais e a meta refere-se à média dos atendimentos de uma gestante durante todo o pré-natal. Para o cálculo é observado que as gestantes chegam na unidade básica de saúde depois do primeiro mês de gestação, por isso, o acompanhamento dura no máximo oito meses. $D_{1}$ é dado pela Equação 5 .

$$
D_{1}=\frac{\frac{\text { numeroGestantesCadastradasEquipe } \times \text { meta }}{8.0}}{2.0}
$$


$D_{2}$ (Equação 6) é a demanda de atendimentos para a Prevenção do Câncer Ginecológico. Considera o custo de um atendimento de 20 minutos (três atendimentos em um instante $k$ ) e visa estipular o número de exames preventivos a ser ofertado. A meta é relacionada com a cobertura desejada em relação à variável.

$$
D_{2}=\frac{\text { populacaoFemininaCadCom } 15 \text { AnosOuMais } \times \frac{\text { meta }}{100}}{3.0}
$$

Todos os cálculos para as demandas de cada um dos serviços listados na Tabela 1 pode ser consultado em [Veras 2015].

Tabela 1. Serviços ofertados, profissionais envolvidos e custos de execução.

\begin{tabular}{l|c|c|c|c}
\hline Serviço Ofertado & Enfermeiro & Médico & Odontólogo & Custo (minutos) \\
\hline Atendimento de Pré-natal & $\mathrm{X}$ & $\mathrm{X}$ & & 30 \\
\hline Prevenção do câncer ginecol. & $\mathrm{X}$ & & & 20 \\
\hline Visitas domiciliares & $\mathrm{X}$ & $\mathrm{X}$ & & 60 \\
\hline Consulta de puericultura & $\mathrm{X}$ & $\mathrm{X}$ & & 30 \\
\hline Consulta médica & & $\mathrm{X}$ & & 15 \\
\hline Consulta de Hipert. e Diabéticos & $\mathrm{X}$ & $\mathrm{X}$ & & 15 \\
\hline Ação col. (escov. dental superv.) & & & $\mathrm{X}$ & 20 \\
\hline Consulta Odontológica & & & $\mathrm{X}$ & 20 \\
\hline Consulta Odontol. à gestante & & & $\mathrm{X}$ & 20 \\
\hline Consulta de enfermagem & $\mathrm{X}$ & & & 30 \\
\hline Vigilância (tubercul. e hansen.) & $\mathrm{X}$ & $\mathrm{X}$ & & 30 \\
\hline
\end{tabular}

\section{O estudo sobre o uso da ferramenta FoxSaúde}

A ferramenta FoxSaúde [Veras et al. 2018] usa uma abordagem baseada em um sistema multiagente para melhorar o desempenho de equipes de saúde por meio do uso de técnicas de inteligência computacional. Além disso, possui uma arquitetura interoperável capaz de simular a executar agendas dinâmicas em diferentes ambientes de atendimento. Por esses motivos, foi usada para incorporar a abordagem de balanceamento de metas proposta neste trabalho. Nesta seção, descreve-se (a) como foi formulada uma estratégia para balanceamento de metas para indicadores de saúde; (b) um ambiente para simulação de atendimentos e (c) os resultados alcançados.

\subsection{Abordagem para o balanceamento automático de metas}

Um balanceamento de metas corresponde a um replanejamento automático das metas, sem fugir da configuração original desejada, objetivando melhorar a aproximação dos indicadores em relação ao valor planejado pela equipe diante de cenários inconstantes. Informalmente, a abordagem cria um "banco de instantes" para cada indicador de um mesmo grupo de profissionais, considerando os instantes abaixo e acima da meta somado às "folgas" planejadas. Entende-se como "folga planejada" um dado instante $k$ no qual, intencionalmente, nenhum serviço foi alocado. Esse banco é redistribuído entre os indicadores do mesmo grupo de profissionais, balanceando-os. Um balanceamento eficiente necessita calcular: (1) Fator de risco de cada indicador;(2) Fator de justiça para "retirar" instantes do indicador apropriado e "dar" o máximo de instantes possível ao indicador que mais necessita. 
Sobre a redistribuição de instantes, temos: no pior caso os instantes abaixo da meta são maiores que os instantes acima somando com as folgas planejadas e, no melhor caso, o valor dos instantes acima da meta com as folgas planejadas é superior ao valor dos instantes abaixo da meta. O objetivo é buscar o balanceamento justo dos indicadores de maneira que fiquem o mais próximo possível da meta original. Neste sentido, se não há indicadores abaixo da meta, então não há balanceamento.

Formalmente, o balanceamento das metas inicia-se a partir de algumas definições. Dado que: $N P s$ indica o número de diferentes profissionais que executam serviços de um mesmo grupo de indicadores, $F P^{P_{x}}$ a quantidade de folgas planejadas para um profissional específico $P_{x} \in E, V_{p}^{I_{i}}$ o volume de trabalho planejado para o indicador $I_{i} \in I, V_{r}^{I_{i}}$ o volume de trabalho realizado para o indicador $I_{i} \in I, D^{I_{i}}$ a demanda de trabalho (meta da equipe) do indicador $I_{i} \in I, F R^{I_{i}}=1-\frac{V_{r}^{I_{i}}}{V_{p}^{I_{i}}}$ o fator de risco do indicador $I_{i} \in I, S I^{I_{i}}=\left(V_{r}^{I_{i}}-D^{I_{i}}\right)$ é o saldo imediato de instantes do indicador $I_{i} \in I$ em relação à demanda planejada pela equipe, $S H^{I_{i}}=S I^{I_{i}}-\left(S I^{I_{i}} \times F R^{I_{i}}\right)$ é o saldo de instantes do indicador $I_{i} \in I$ considerando os riscos para a ocorrência de eventos, $N I^{I_{i}}=\left(D^{I_{i}}-V_{r}^{I_{i}}\right)$ é a necessidade imediata de instantes do indicador $I_{i} \in I$ em relação à demanda, $N H^{I_{i}}=N I^{I_{i}}+\left(N I^{I_{i}} \times F R^{I_{i}}\right)$ é a necessidade de instantes do indicador $I_{i} \in I$ considerando os riscos para ocorrência de eventos, $D I n d^{+}$a quantidade de indicadores acima da meta obtido por um grupo de $N P s$ profissionais e $D I n d^{-}$a quantidade de indicadores abaixo da meta obtido por um grupo de $N P s$ profissionais. A partir disso, o banco de instantes de um grupo formado por NPs profissionais pode ser calculado por:

$$
B H^{*}=\sum_{i=1}^{N P s} F P^{P_{i}}+\sum_{j=1}^{D I n d^{+}} S H^{I_{j}}
$$

Desse modo, para a retirada justa de instantes "extras" do volume dos indicadores a serem planejados para o mês subsequente, é necessário considerar apenas o somatório dos saldos e desconsiderar as folgas planejadas, pois elas serão usadas no replanejamento dos indicadores abaixo da meta. Com isso, o replanejamento de um indicador qualquer pertencente a um grupo de profissionais é feito a partir das definições a seguir. Seja: $S H^{*}=B H^{*}-\sum_{i=1}^{N P s} F P^{P_{i}}$ o saldo de instantes do grupo dos $N P s$ profissionais, $F J_{I_{i}}^{+}=$ $\frac{S H^{I_{i}}}{S H^{*}}$ o fator de justiça para o indicador $I_{i} \in I$ acima da meta e $N H^{*}=\sum_{x=1}^{D I n d^{-}} N H^{I_{x}}$ a necessidade de instantes do grupo dos NPs profissionais. O valor da nova demanda para o planejamento do mês subsequente de um indicador acima da meta é dado por:

$$
R P_{I_{i}}^{+}=\left\{\begin{array}{l}
D^{I_{i}}+\left(D^{I_{i}} \times F R^{I_{i}}\right) \text { se } N H^{*}=0 \\
D^{I_{i}}-\left(N H^{*} \times F J_{I_{i}}^{+}\right)+\left(S H^{I_{i}} \times F R^{I_{i}}\right) \text { se } N H^{*}>0 \\
D^{I_{i}}-\left(D^{I_{i}} \times F R^{I_{i}}\right) \text { se } B H^{*} \leq N H^{*}
\end{array}\right\}
$$

Semelhantemente, a distribuição dos instantes do banco de instantes $\left(B H^{*}\right)$ entre os indicadores abaixo da meta deve ser realizada de maneira justa. Para tanto, o replanejamento de um indicador que necessita de instantes para aproximar-se da meta (ou superá-la) é feito partindo do cálculo do fator de justiça para o recebimento justo de instantes, dado 
por:

$$
F J_{I_{i}}^{-}=\frac{N H^{I_{i}}}{N H^{*}} \text { o fator de justiça para o indicador } I_{i} \in I \text { abaixo da meta. }
$$

Logo, o valor da nova demanda para o planejamento do mês seguinte de um indicador abaixo da meta é dado por:

$$
R P_{I_{i}}^{-}=\left\{\begin{array}{l}
D^{I_{i}}+\left(B H^{*} \times F J_{I_{i}}^{-}\right) \text {se } N H^{*}>B H^{*} \\
D^{I_{i}}+N H^{I_{i}} \text { se } N H^{*} \leq B H^{*}
\end{array}\right\}
$$

Embora as novas demandas tenham sido definidas, ainda faz-se necessário estipular um limite máximo (Demanda mínima $=D^{I_{i}}+\left(D^{I_{i}} \times F R^{I_{i}}\right)$ ) tanto para retirada quanto para a distribuição de instantes dos indicadores de forma a não permitir que aconteçam desvios discrepantes em relação ao planejamento inicial. Esse limite está associado com os riscos dos eventos e é calculado como uma demanda mínima aceitável. Assim sendo, a nova demanda para os indicadores (acima ou abaixo da meta) é limitada da seguinte forma:

$$
R P_{I_{i}}^{+} \mid R P_{I_{i}}^{-}=\left\{\text {Demanda mínima se }\left(R P_{I_{i}}^{+} \mid R P_{I_{i}}^{-}\right)<\text {Demanda mínima }\right\}
$$

Com a realização dos ajustes automáticos para as demandas, é possível recalcular e replanejar (mensalmente) as metas por meio das fórmulas estabelecidas na Seção 3.2.

\subsection{Ambiente para simulações}

A alocação de um serviço em uma agenda caracteriza um atendimento quando o serviço for efetivamente executado em um ambiente de atendimento qualquer e um ambiente virtual de atendimentos em saúde deve simular a execução dos serviços contidos em uma agenda mensal. FoxSaúde considera duas estruturas idênticas de agendas para representar, respectivamente, as agendas planejadas e os atendimentos simulados. O ambiente criado permite a ocorrência de eventos negativos que atrapalham a execução das agendas planejadas e possui uma estrutura para indicar o exato momento (semana, dia e instante $k$ ) dos acontecimentos. A Figura 1 exemplifica a ocorrência de um evento e demonstra como acontece o desvio em um serviço planejado. No exemplo de um atendimento realizado conforme o planejamento, um serviço com $i d=3$ foi alocado na agenda planejada e também foi alocado na agenda simulada. No outro exemplo destacado pela figura, o serviço com $i d=7$ foi alocado na agenda planejada, porém, na agenda simulada foi alocado um outro com $i d=2$, registrando a ocorrência de um desvio na execução do serviço originalmente planejado. Neste caso, foi executado um "serviço alternativo".

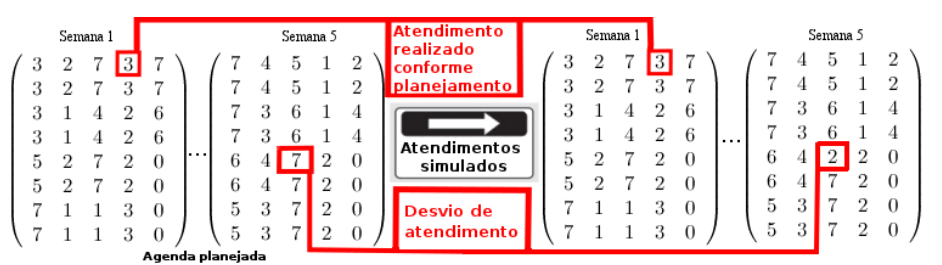

Figura 1. Exemplo de desvio na execução de um atendimento planejado. 
É possível também simular a ocorrência de eventos associados à cada serviço ofertado para, durante os atendimentos, dispará-los aleatoriamente ou estocasticamente, dependendo do serviço planejado para o instante $k$ e do cenário desejado. Cada transição do ambiente marca uma evolução da simulação de um momento $m$, constituído por instantes $k$, dias e semanas. Na Tabela 2 é possível notar um modelo de instância usado para simular o cenário de uma unidade básica de saúde composta por suas variáveis sociais (ou populacionais) e as respectivas metas associadas a cada serviço ofertado (listagem da Tabela 1). Para as simulações foram considerados como recursos humanos disponíveis dois enfermeiros, dois médicos e três odontólogos.

Tabela 2. Modelo de instância para Unidade Básica de Saúde

\begin{tabular}{|c|c|c|}
\hline Tipo & Descritor & Valor \\
\hline \multirow[t]{11}{*}{ Variável populacional } & numeroGestantesCadastradasEquipe & 40 \\
\hline & populacaoFemininaCadastradaCom15AnosOuMais & 321 \\
\hline & numeroMenoresDeDoisAnos & 15 \\
\hline & numeroDeMenoresDeUmAnoAcompanhadas & 09 \\
\hline & numeroDePessoasAcompanhadasNoDomicilio & 32 \\
\hline & numeroDeMenoresDeCincoAnosCadastradas & 28 \\
\hline & numeroDiabeticosCadastrados & 34 \\
\hline & numeroHipertensosCadastrados & 40 \\
\hline & populacaoCadastrada & 3200 \\
\hline & numeroPessoasComTuberculoseCadastradas & 10 \\
\hline & numeroPessoas ComHanseniaseCadastradas & 22 \\
\hline \multirow[t]{11}{*}{ Meta } & Média de consultas durante o pré-natal & 12 \\
\hline & Cobertura para prevenção do câncer ginecológico & $30 \%$ \\
\hline & Cobertura para demanda de visitas domiciliares & $70 \%$ \\
\hline & Média de consultas de puericultura & 3 \\
\hline & Cobertura para demanda de consultas médica & $18 \%$ \\
\hline & Média de consultas de Hipertensos e Diabéticos & 2 \\
\hline & Cobertura para demanda de ação coletiva de escovação dental & $18 \%$ \\
\hline & Cobertura para demanda de consultas odontológicas & $25 \%$ \\
\hline & Cobertura para demanda de consultas odontológicas às gestantes & $100 \%$ \\
\hline & Cobertura para demanda de consultas de enfermagem & $6 \%$ \\
\hline & Média de consultas para vigilância (tuberculose e hanseníase) & 2 \\
\hline
\end{tabular}

\subsection{Resultados e discussões}

Na Figura 2 (esq.) é possível visualizar um exemplo do monitoramento exercido pela ferramenta sobre o indicador associado às consultas de puericultura, executado durante a evolução do ambiente com um ciclo de 12 meses para o Cenário 2 (C2) no grupo de testes com serviços alternativos. O gráfico revela que o planejamento manteve-se estável durante praticamente todo o ciclo evolucionário. Como os eventos acontecem estocasticamente as influências negativas variaram durante o ciclo e em alguns momentos (correspondente a 4 meses) o indicador não possuiu seu valor estabilizado acima da meta, mesmo com os serviços alternativos sendo executados. No mês 2 , observa-se uma pequena elevação no planejamento, em uma tentativa da ferramenta para encontrar melhores agendas. Não coincidentemente, no mesmo mês, o valor simulado do indicador também obteve um valor crescente. Esses dois fatores evidenciam que os indicadores sofrem influências tanto do planejamento quanto dos eventos negativos.

Na Figura 2 (dir.) é mostrado o monitoramento com a ferramenta balanceando automaticamente as metas. Em apenas um dos meses da evolução dos atendimentos o indicador foi valorado abaixo da meta, evidenciando que o planejamento foi consistente 
Figura 2. Monitoramento de puericultura com serviços alternativos em C2 (esq). Monitoramento com serviços alternativos e replanejamento de metas em C2 (dir.)
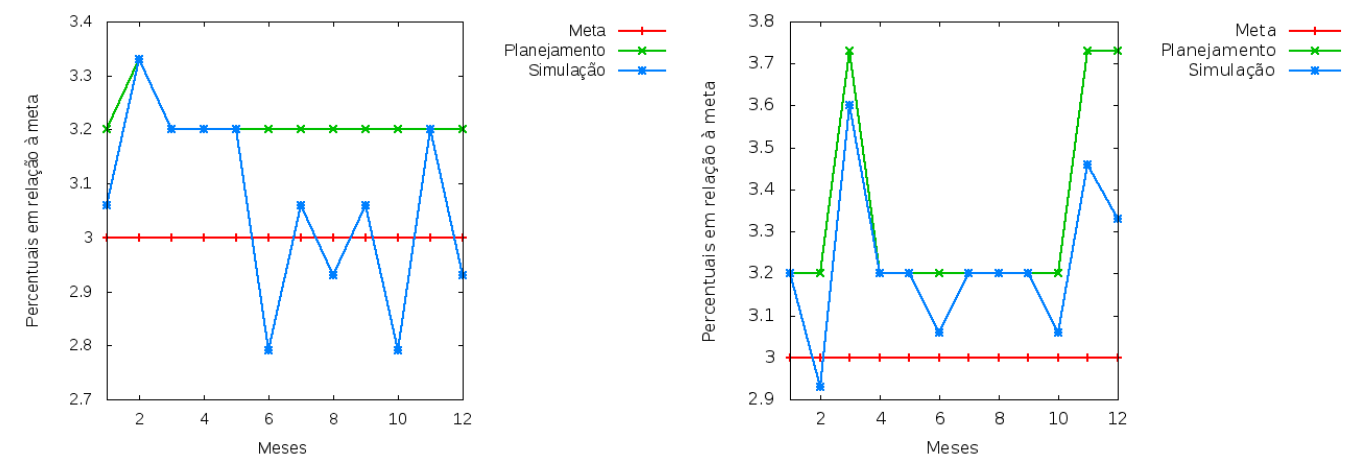

em manter o indicador acima da meta mesmo diante das influências negativas exercidas pelos eventos. FoxSaúde dotado do balanceamento automático percebe que os riscos no final da simulação (ciclo de um mês) crescem e decide sobre um aumento no planejamento para prevenir queda do indicador. O comportamento dos indicadores ao final das simulações pode ser visualizado por meio da Tabela 3, bem como, o $p$-valor do teste estatístico de $t$-student sobre o percentual dos serviços executados, que mede a influência dos eventos negativos sobre o planejamento. A escolha desse teste deu-se a partir dos resultados positivos sobre os testes de normalidade para a distribuição do percentual de serviços executados em toda a amostra em relação à sua média. A análise feita procu-

Tabela 3. Dados estatísticos da simulação (sem balanceamento)

\begin{tabular}{|c|c|c|c|c|c|c|c|}
\hline \multirow[b]{2}{*}{ Cenário } & \multirow{2}{*}{$\begin{array}{c}\text { Serviços } \\
\text { Alternativos }\end{array}$} & \multicolumn{2}{|c|}{ Indicadores } & \multicolumn{4}{|c|}{ Serviços executados } \\
\hline & & DP & Variância & Média (\%) & DP & Variância & $p$-valor \\
\hline \multirow[t]{2}{*}{$\mathrm{C} 1$} & Sem & 0.84 & 0.71 & 23.1 & 1.78 & 3.17 & 0.8053 \\
\hline & Com & 0.95 & 0.92 & 21.6 & 2.03 & 4.12 & 0.8131 \\
\hline \multirow[t]{2}{*}{$\mathrm{C} 2$} & Com & 0.91 & 0.83 & 97.0 & 0.57 & 0.33 & 0.6826 \\
\hline & Sem & 0.91 & 0.83 & 94.8 & 0.69 & 0.48 & 0.8809 \\
\hline
\end{tabular}

rou atestar a semelhança estatística dos resultados gerados pelas simulações em relação à influência dos eventos negativos no planejamento, mostrando que não há alterações relevantes entre os dados da amostra (trinta execuções da simulação) no tocante à média dos serviços executados. É possível concluir que os dados da amostra (trinta execuções) das simulações em cada um dos dois cenários são estatisticamente iguais com significância de $95 \%$ podendo atingir uma probabilidade de $88,09 \%$ para o valor da média dos serviços executados ser de fato o valor encontrado pelas simulações, como foi o caso do cenário 2 sem serviços alternativos.

Os gráficos da Figura 2 e mostram um certo grau de variação dos indicadores em função da evolução do ambiente, principalmente, por conta da influência negativa com características estocásticas sofrida por meio dos eventos que "desviam" a execução do planejamento. Os dados do monitoramento dos indicadores nos dois cenários, enumerados conforme a Tabela 4, comprovam as características estocásticas para a ocorrência dos eventos durante as simulações por meio da alta variância dos indicadores acima e abaixo da meta. Fato que não ocorreu no cenário de controle por conta não influência no planejamento e balanceamento das metas. O aumento do tempo da simulação não 
Tabela 4. Dados estatísticos da simulação (sem balanceamento).

\begin{tabular}{|c|c|c|c|c|c|c|c|}
\hline \multirow[b]{2}{*}{ Cenário } & \multirow{2}{*}{$\begin{array}{c}\text { Serviços } \\
\text { Alternativos }\end{array}$} & \multicolumn{3}{|c|}{$\begin{array}{c}\text { Indicadores } \\
\text { acima da meta }\end{array}$} & \multicolumn{3}{|c|}{$\begin{array}{c}\text { Indicadores } \\
\text { abaixo da meta }\end{array}$} \\
\hline & & Média & $\mathbf{D P}^{3}$ & Variância & Média & DP & Variância \\
\hline \multirow[t]{2}{*}{$\mathrm{C} 1$} & Sem & 34.7 & 2.02 & 4.11 & 97.2 & 1.99 & 3.97 \\
\hline & Com & 37.2 & 2.15 & 4.63 & 91.4 & 1.80 & 3.24 \\
\hline \multirow[t]{2}{*}{$\mathrm{C} 2$} & Com & 92.9 & 4.04 & 16.68 & 36.8 & 2.99 & 8.64 \\
\hline & Sem & 75.5 & 4.25 & 18.11 & 53.9 & 3.33 & 10.75 \\
\hline
\end{tabular}

promoveu nenhuma alteração estatística em relação a média do percentual dos serviços executados. Isso implica que a quantidade de serviços executados em cada cenário é praticamente a mesma em toda a amostra, assim, as variações se dão nas execuções dos serviços ofertados. Esse comportamento certifica o padrão na evolução do ambiente simulado nos cenários descritos quanto à ocorrência dos eventos e solidifica o comportamento estocástico desses eventos em relação à influência negativa dos indicadores, pois a primitiva básica dos indicadores (os serviços alocados nas agendas) sofre desvios não determinísticos. A Tabela 5 mostra um comparativo do ganho médio (em percentual) dos

Tabela 5. Ganho médio em percentual da simulação (com balanceamento).

\begin{tabular}{c|c|c|c}
\hline \multirow{2}{*}{ Cenário } & $\begin{array}{c}\text { Serviços } \\
\text { Alternativos }\end{array}$ & Indicadores acima da meta & Indicadores abaixo da meta \\
\hline \multirow{2}{*}{ C2 } & Com & $+78.27 \%$ & $-41.34 \%$ \\
\cline { 2 - 4 } & Sem & $+120.26 \%$ & $-35.34 \%$ \\
\hline
\end{tabular}

indicadores obtidos a partir do uso da ferramenta no cenário 2 (C2) relação aos resultados apresentados pelas Tabelas 3 e 4 (sem o uso da ferramenta). No cenário de controle (C1) não foram encontradas variações entre os resultados com e sem o uso do balanceamento. Nste cenário, a proposta não exerceu nenhuma influência em relação ao equilíbrio dos indicadores em função de suas metas, comportamento esse, harmônico com os objetivos propostos para o cenário. No cenário 2 (C2), a atuação da ferramenta melhorou a evolução e manutenção dos indicadores em relação à sua meta, segundo a Tabela 5. Com os resultados, é possível assegurar que o comportamento estatístico da abordagem é o mesmo com e sem o uso do balanceamento, garantido sua eficácia a partir da interferência da ferramenta no equilíbrio dos indicadores em relação às metas da equipe. Como a abordagem possui o mesmo comportamento, então o aumento dos indicadores acima da meta e a redução dos indicadores abaixo da meta são por conta da intervenção do balanceamento proposto. $\mathrm{O}$ resultado mostrou-se estatisticamente eficiente na melhora dos valores para indicadores de saúde em um cenário hostil que exerce influências negativas sobre a valoração dos mesmos.

\section{Considerações Finais}

Os testes da abordagem proposta enumeraram resultados satisfatórios que fortalecem o uso da orientação por metas como estratégia de planejamento de atendimentos. Mostraram que apenas o planejamento orientado por metas não é eficaz para manter os indicadores equilibrados diante da ocorrência de eventos negativos em cenários com longos ciclos de duração. Sobre isso, os resultados do uso do balanceamento automático de metas ofereceram dados estatísticos positivos a uma significância de 95\% para o cenário analisado, 
mostrando que um replanejamento com balanceamento de metas pode aproximar os indicadores dos valores desejados. Para garantir a replicabilidade desta pesquisa, todos os valores associados às variáveis sociais, configurações e parametrização dos algoritmos utilizados podem ser visualizados em [Veras 2015].

Alguns fatores podem causar viés nesta pesquisa, por exemplo, a elaboração de um modelo de instância a partir de dados de poucas unidades básicas de saúde. Instâncias heterogêneas permitiriam a realização de simulações mais completas, podendo até simular toda a atenção básica de um município. A falta de planejamento de metas iniciais para a oferta de serviços de saúde pode inviabilizar o uso da proposta. Sugere-se como trabalho futuro um estudo de algoritmos de otimização para gerar metas iniciais para o planejamento de atendimentos em saúde, abreviando essa lacuna da abordagem proposta.

\section{Referências}

BRASIL, M. d. S. (2012). Programa nacional de melhoria do acesso e da qualidade da atenção básica (PMAQ): manual instrutivo. Ministério da Saúde: (Série A. Normas e Manuais Técnicos).

Braun, L., Wiesman, F., Herik, v. d. J., and Hasman, A. (2005). Agent support in medical information retrieval. In Working notes of the IJCAI-05. Workshop on agents applied in health care, pages 16-25.

Cardoso, L. and al, e. (2014). Healthcare interoperability through intelligent agent technology. Procedia Technology, 16:1334-1341.

Isern, D., Sánchez, D., and Moreno, A. (2010). Agents applied in health care: A review. International Journal of Medical Informatics, 79(3):145-166.

Oliveira, É. d. N., Cainelli, J. C., Pilz, C., Cazella, S. C., and Dahmer, A. (2012). Aplicativo auxiliando a melhoria da atenção básica no brasil application to assist in improving of health in brazil's primary care.

Silva, R. C., Forster, A. C., Alves, D., Ferreira, J. B., and Sant'Anna, S. C. (2012). Ferramenta computacional para programa de melhoria da atenção básica (pmaq-ab). In Atas do XIII Congresso Brasileiro de Informática em Saúde.

Veras, N. L. (2015). Planejamento de Atendimentos em Saúde Orientado por Metas com Suporte à Simulação de Eventos Estocásticos Utilizando Agentes Inteligentes. $\mathrm{PhD}$ thesis, Dissertação (Mestrado) Universidade Estadual do Ceará, Mestrado Acadêmico em Ciência da Computação.

Veras, N. L., Silva, E. B. J., and Viana, W. (2018). Foxsaúde: uma abordagem multiagente para otimização da atenção básica. In Anais do XVI Congresso Brasileiro de Informática em Saúde, pages 471-484. SBIS.

Vermeulen, I. B., Bohte, S. M., Elkhuizen, S. G., Lameris, H., Bakker, P. J., and Poutré, H. L. (2009). Adaptive resource allocation for efficient patient scheduling. Artificial intelligence in medicine, 46(1):67-80.

Wiesman, F., Hasman, A., Braun, L., and van den Herik, J. (2006). Information retrieval in medicine: The visual and the invisible (information retrieval im gesundheitswesen: Das visuelle und das unsichtbare). it-Information Technology (vormals it+ ti), 48(1/2006):24-32. 